ARTICLE

DOI: $10.1038 / s 41467-018-05160-1$

\title{
Ratiometric nanothermometer in vivo based on triplet sensitized upconversion
}

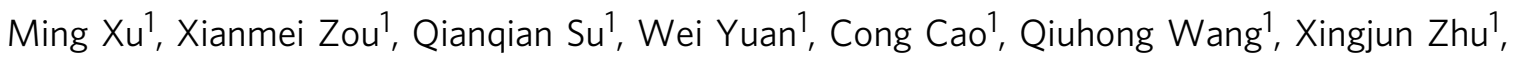
Wei Feng (i) ${ }^{1} \&$ Fuyou $\mathrm{Li}^{1}$

Temperature is an essential factor that counts for living systems where complicated vital activities are usually temperature dependent. In vivo temperature mapping based on noncontact optical approach will be beneficial for revealing the physiological phenomena behind with minimized influence to the organism. Herein, a highly thermal-sensitive upconversion system based on triplet-triplet annihilation (TTA) mechanism is pioneered to indicate body temperature variation sensitively over the physiological temperature range. The temperatureinsensitive $\mathrm{NaYF}_{4}$ : Nd nanophosphors with NIR emission was incorporated into the temperature-responsive TTA-upconversion system to serve as an internal calibration unit. Consequently, a ratiometric thermometer capable of accurately monitoring the temperature changes in vivo was developed with high thermal sensitivity $\left(\sim 7.1 \% \mathrm{~K}^{-1}\right)$ and resolution $(\sim 0.1 \mathrm{~K})$.

\footnotetext{
${ }^{1}$ Department of Chemistry \& State Key Laboratory of Molecular Engineering of Polymers, Fudan University, Shanghai 200433, China. Correspondence and requests for materials should be addressed to W.F. (email: fengweifd@fudan.edu.cn) or to F.L.(email: fyli@fudan.edu.cn)
} 
T he luminescent nanothermometry has received much attention in recent years because it has a broad range of applications involving nanomedicine, microfluidics, nanoelectronics, and integrated photonic devices ${ }^{1-3}$. The development of such highly sensitive nanotermometer is very important in view of its great potential to revolutionize relevant areas, especially in the part of diagnosis and therapy ${ }^{4}$. Recently, temperature monitoring in vivo has been proposed as a useful tool for the studies of physiology, medical diagnosis, and controllable hyperthermia treatment ${ }^{5-10}$. The contactless thermometry based on luminescence imaging provides a noninvasive and observable approach to the mapping of body temperature ${ }^{1-3}$. Notably, upconversion based on the anti-Stokes process that can avoid auto-fluorescence of biological system, is a promising technique for the development of thermometer in vivo ${ }^{11-13}$. In this context, some thermometers were developed based on the lanthanidedoped upconversion nanophosphors (UCNPs). However, the UCNPs-based thermometers generally showed moderate thermal sensitivity $\left(<1.6 \% \mathrm{~K}^{-1}\right)$ and resolution $(>0.5 \mathrm{~K})$, as well as poor luminescence efficiency ${ }^{14-18}$. Therefore, a highly sensitive thermometer capable of monitoring the slight temperature variation in vivo is still urgently needed.

As the most effective anti-Stokes process, upconversion based on triplet-triplet annihilation (TTA) is potentially thermosensitive ${ }^{19-23}$. TTA-upconversion involves multiple energy transfer in the annihilator and sensitizer dyad. Intuitively, TTAupconversion requires diffusion of the component chromophores, which is sensitive to small temperature changes ${ }^{24-30}$. Nevertheless, the example of thermometer in vivo based on the TTAupconversion technique has not been reported, which is hampered by significant challenges such as irregular temperature response or low thermal sensitivity in the physiological circumstance, and serious concentration dependence.

Herein, we designed an optimized TTA dyad to explore thermometry in vivo. At higher temperature, the improvement in diffusion rate and collision probability of the chromophores led to sharply enhanced TTA-upconversion ${ }^{31-33}$. Together with the suppression of nonradiative deactivation in our design, the upconversion luminescence (TTA-UCL) was positively temperature-dependent over the physiological temperature range (Supplementary Figure 1). In order to minimize influence from biological environment and to enable concentration-independent output of indicating signals, the TTA dyad was encapsulated with a thermal-insensitive internal standard (Supplementary Figure 2) ${ }^{34}$. The reference showed NIR emission in the second biological window, which was also applicable for bioimaging ${ }^{35,36}$. Consequently, a ratiometric thermometer was achieved as TTA-Nd-NPs (Fig. 1). The potential use of TTA-Nd-NPs for ratiometric thermometry in vivo was demonstrated by the accurate measurement of temperature distributions in tissue, and the detection of temperature changes in mice caused by inflammation. The work makes great sense for a broad research areas of upconversion, thermometry, nanomedicine, and life science.

\section{Results}

Upgrades to TTA system: deactivation suppression. In terms of screening a possible TTA system for temperature indication, the photo-stable and effective BODIPY \& PtTPBP dyads are promising candidates ${ }^{37-40}$. Apparently, phenyl ring at the top of conventional BODIPY annihilators (e.g., BD) could freely rotate to dissipate energy, thus leading to pronounced thermal deactivation (Fig. 2a $)^{41}$. Diffusion enhancement at higher temperature is a positive factor for the TTA-upconversion process (Supplementary Figure 1), while thermal deactivation is a negative factor. The competitive effect between deactivation and diffusion factors

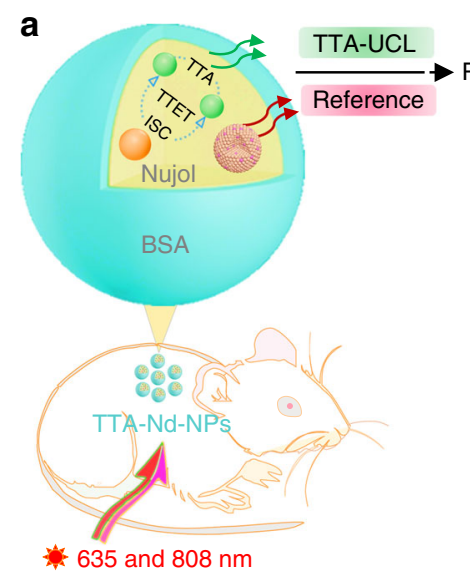

Ratiometric thermometry

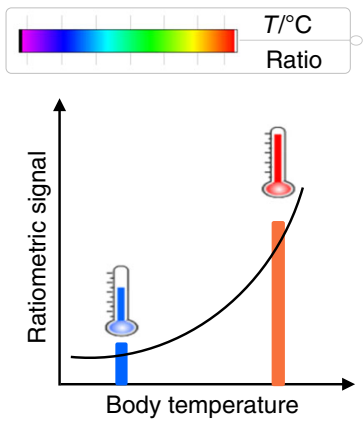

b
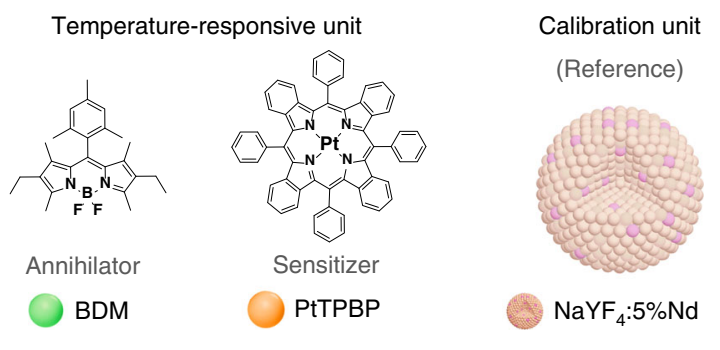

Fig. 1 Schematic illustration of the TTA-Nd-NPs based ratiometric thermometry in vivo. a TTA-upconversion is sensitively responsive to temperature changes. With the assistance of an internal reference, the calibrated TTA-UCL signals become capable for accurate temperature monitoring in a small animal. $\mathbf{b}$ Chemical structures of the TTA chromophores containing BDM (TTA annihilator) and PtTPBP (TTA sensitizer), and schematic structure of the $\mathrm{Nd}^{3+}$ nanophosphor (reference). BSA: bovine serum albumin, ISC: intersystem crossing, TTET: triplet-triplet energy transfer, TTA: triplet-triplet annihilation

would probably result in irregular thermal responses of the TTA system. As shown in Fig. 2b, a positive-to-negative transition at $40{ }^{\circ} \mathrm{C}$ was indicated with week TTA-UCL signal for BD \& PtTPBP in nujol solvent. The TTA-UCL enhanced by positive diffusion effect was even overwhelmed by the negative quenching effect at higher temperature, which caused opposite thermalresponse over $40^{\circ} \mathrm{C}$. Due to the quenching effect from thermal deactivation, the development of a sensitive TTA-thermometer in vivo is still challenging.

We took a simple but effective strategy to mitigate deactivation effect by using the rotation-suppressed BDM as the annihilator (Supplementary Figure 3). The structural modification had minor influence on the UV-Vis absorption, whereas it enabled a 1.6-fold improvement of the fluorescence (Supplementary Figure 4). Moreover, a remarkable mitigation in the thermal deactivation of fluorescence was observed (Fig. 2a), which was equivalent to the amplification of positive diffusion effect. Notably, our design pushed the limit of thermosensitive TTA system where phase transition or polymer-chain softening was generally required to enhance the diffusion effect $24,28,29,33$. Consequently, for the BDM \& PtTPBP directly in liquid solvent, a continuous enhancement of TTA-UCL was achieved from 10 to $60^{\circ} \mathrm{C}$ (Fig. 2b). Therefore, the discovery provided a new frontier in temperature-enhanced TTA system.

Upgrades to TTA system: ratiometric calibration. The thermal response of BDM \& PtTPBP over physiological temperature range is highly desirable. Nevertheless, calibration of TTA-UCL is 
a

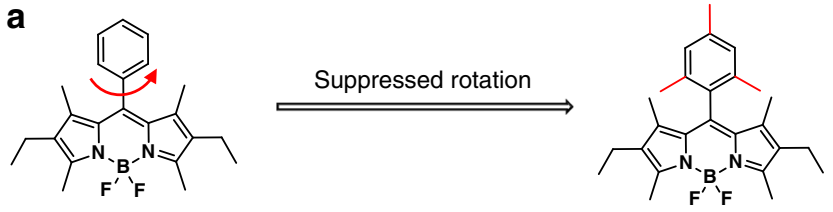

$\mathrm{BD} \longrightarrow$ Mitigated thermal deactivation $\longrightarrow$ BDM
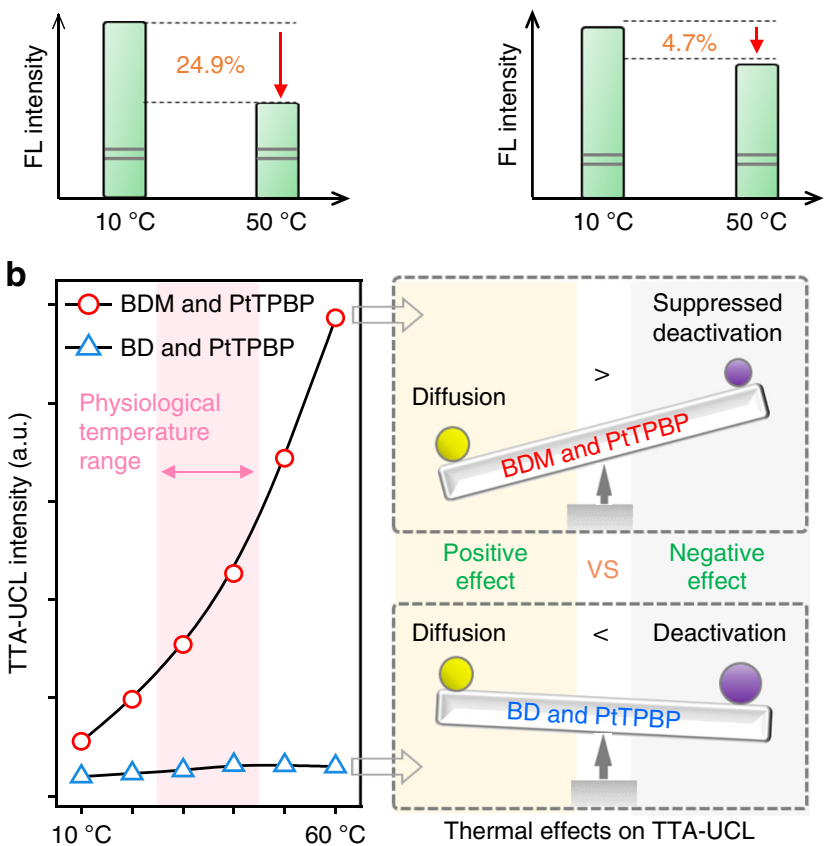

Fig. 2 Strategy for achieving highly positive thermal response in a TTA system. a The structure modification and thus suppressed thermal deactivations of fluorescence. $\mathbf{b}$ The temperature responses of TTA-UCL signals emitted from BDM \& PtTPBP and BD \& PtTPBP, respectively. When negative deactivation effect is suppressed, the positive diffusion effect becomes more powerful to give a positive temperature-response trend with high sensitivity. A $635 \mathrm{~nm}$ laser $\left(100 \mathrm{~mW} \mathrm{~cm}^{-2}\right)$ was used as the excitation source. The concentrations of BDM, BD, PtTPBP, and L-ascorbyl 6-palmitate in nujol solvent were $1 \times 10^{-3} \mathrm{M}, 1 \times 10^{-3} \mathrm{M}, 5 \times 10^{-5} \mathrm{M}$ and $1 \mathrm{mg} \mathrm{mL}^{-1}$, respectively

still necessary for thermometry in a complicated biosystem ${ }^{42}$. Therefore, a calibration unit was introduced into the TTA system in the present study. The TTA-Nd-NPs material was prepared through self-assembly of BDM \& PtTPBP and $\beta-\mathrm{NaYF}_{4}: 5 \% \mathrm{Nd}$ (Supplementary Figure 5) in a high pressure homogenizer. The calibration signal $\left({ }^{4} \mathrm{~F}_{3 / 2}-{ }^{4} \mathrm{I}_{11 / 2}\right.$ of $\left.\mathrm{Nd}^{3+}\right)$ had minimized overlap with the TTA-UCL, which could avoid the crosstalk effect (Supplementary Figure 2). The nanocomposite structure of organic-inorganic TTA-Nd-NPs was revealed in the transmission electron microscope images with a diameter of $\sim 160 \mathrm{~nm}$ (Supplementary Figure 6). The hydrodynamic diameter of TTA-NdNPs was $165 \mathrm{~nm}$, and the size kept unchanged within $10-50^{\circ} \mathrm{C}$ (Supplementary Figure 7). In addition, no obvious toxicity of TTA-Nd-NPs material was revealed in the biocompatibility study (Supplementary Figure 8).

The TTA dyad BDM \& PtTPBP in TTA-Nd-NPs gave rise to a green upconversion emission with lifetime of $170 \mu$ s (Supplementary Figure 9) and absolute quantum efficiency of $3.1 \%$ at room temperature (see Methods for details). A sustained downward trend in TTA-UCL intensity was observed as the TTA-Nd-NPs returned to room temperature through natural cooling, while the TTA-UCL intensities of control groups with constant temperatures remained unchanged all along. (Supplementary Figure 10). Moreover, the thermal sensitivity of TTA-
UCL was easily controllable by regulating the concentration ratio of sensitizer (Supplementary Figure 11). A quadratic-to-linear dependence between TTA-UCL intensity and $635 \mathrm{~nm}$ excitation power density was clearly observed, and the threshold excitation intensity was revealed to be $65 \mathrm{~mW} \mathrm{~cm}^{-2}$ (Fig. 3a) ${ }^{43}$. Under lowpower excitation below the threshold, triplets of the annihilators decay spontaneously to result in a quadratic dependence, which is a common phenomenon in the bimolecular TTA-upconversion system $^{43}$. In addition, the luminescence intensity of $\mathrm{Nd}^{3+}$ nanophosphors in TTA-Nd-NPs was linear dependent on the $808 \mathrm{~nm}$ excitation power density (Fig. 3a) with an absolute quantum

efficiency of $10.2 \%$ (see Methods for details). In the following experiments, the power densities of coupled 635 and $808 \mathrm{~nm}$ excitation light were therefore set beyond the threshold ( $>65$ $\mathrm{mW} \mathrm{cm} \mathrm{cm}^{-2}$ ) to ensure both of the luminescence processes in a linear regime. Herein, laser power density in the range of $100-200 \mathrm{~mW} \mathrm{~cm}^{-2}$ was an optimal choice for excitation, which could enable power-independent signal output in the lowpower region (Fig. 3a). With all above upgrades to TTA system, problems from irregular (or insensitive) temperature response and concentration dependence are solved in theory, and the concept of ratiometric probe based on TTA system is constructed.

Ratiometric response of TTA-Nd-NPs. Then, the thermosensitive properties of TTA-Nd-NPs were accurately investigated. The luminescence spectra of TTA-Nd-NPs were measured in a dual-channel test system as illustrated in Fig. 3b. In the measurement, the sample temperature was precisely controlled $( \pm 0.1$ $\mathrm{K})$ with a thermocouple placed in the solution of sample rather than around the surface of cuvette (Supplementary Figure 12). As the temperature increases, the TTA-UCL intensity enhanced rapidly to show a quadratic tendency (Fig. 3c). The calculated thermal sensitivity of TTA-UCL in the TTA-Nd-NPs $\left(\sim 12 \% \mathrm{~K}^{-1}\right)$ was far better than that of the reported thermosensitive $\mathrm{NaYF}_{4}$ : $\mathrm{Yb}, \mathrm{Er} @ \mathrm{NaLuF}_{4} \mathrm{UCNP}\left(\sim 0.8 \% \mathrm{~K}^{-1}\right)$ within $10-50{ }^{\circ} \mathrm{C}$ (Supplementary Figure 13$)^{17}$. In contrast, the emission from $\mathrm{Nd}^{3+}$ nanocrystals in TTA-Nd-NPs slowly declined with a slop less than $0.03 \% \mathrm{~K}^{-1}$ (Fig. 3c). Indeed, the $\mathrm{Nd}$ ion was not good for thermal response due to its low thermal sensitivity ${ }^{44,45}$. Attributed to the highly thermosensitive nature of our TTA system that was served as temperature-responsive unit, herein the thermal insensitive $\mathrm{Nd}^{3+}$ nanocrystal could be designed to just serve as an internal standard. The absorption/emission of $\mathrm{Nd}^{3+}$ nanocrystals showed no overlap with that of the TTA system, which enabled the unaffected performance of TTA system. Actually, calibration unit for the TTA system can also be upgraded in the future, for example employing other rare earth ions based core-shell nanoparticles that showed markedly thermal sensitivity, which is possibly beneficial for achieving a better performance in ratiometric thermometry 46,47 .

In the biological system, the luminescence of exogenous probes generally depends on the specific bio-distributions (Supplementary Figure 14). Herein, the strategy of ratiometric nanocapsule was appropriately used to address this issue. For each individual TTA-Nd-NPs capsule, theoretically the luminescence ratio of TTA dyad and $\mathrm{Nd}^{3+}$ nanocrystal maintains the same, ensuring the concentration-independent output ${ }^{1-3}$. The calibrated TTAUCL signal output (ratio of $I_{\text {TTA-UCL }} / I_{\text {Reference }}$ ) did not change with the concentration variations from the initial material $(15 \mathrm{mg}$ $\left.\mathrm{mL}^{-1}\right)$ to a very dilute solution $\left(0.9 \mathrm{mg} \mathrm{mL}^{-1}\right)$, even though the TTA-UCL signals were much decreased to show an obviously concentration-dependent signal output (Fig. 3d). The observations clearly demonstrated the importance of ratiometric probing 


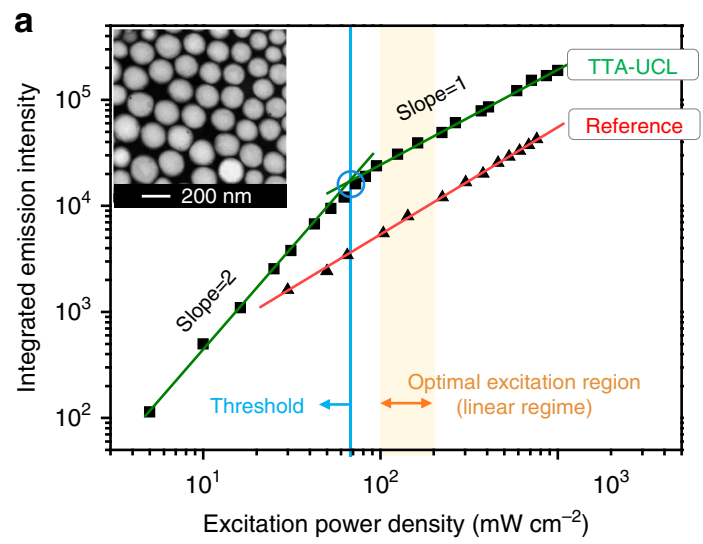

b
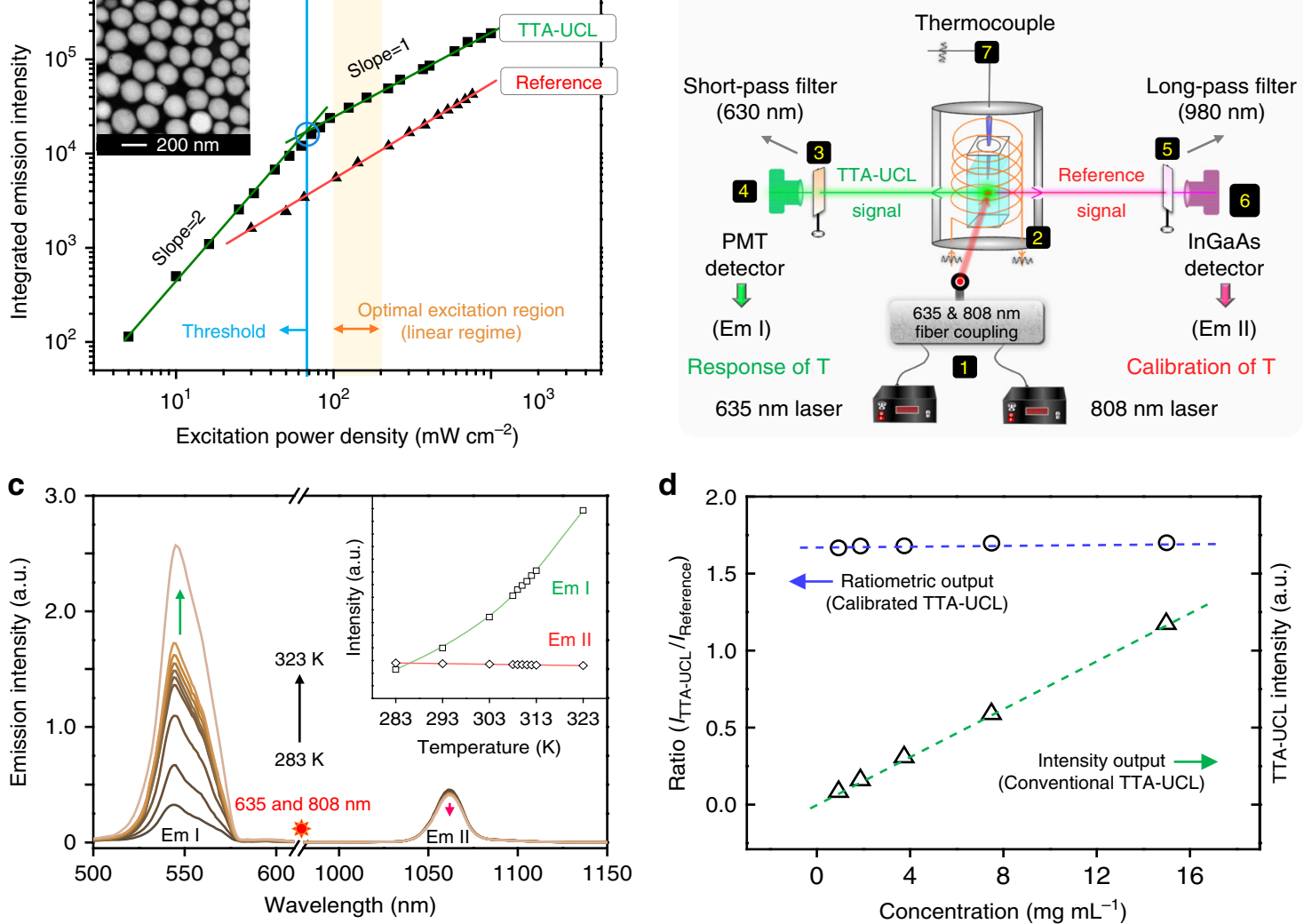

Fig. 3 Properties investigation of TTA-Nd-NPs. a The dual-emissive properties of TTA-Nd-NPs at varying excitation power. TTA-UCL signal peaked at 540 $\mathrm{nm}$ and Reference signal peaked at $1060 \mathrm{~nm}$ were plotted in the emission profiles. Inset: the transmission electron microscope image of TTA-Nd-NPs after negative staining with sodium phosphotungstate. Scale bar is $200 \mathrm{~nm}$. b Schematic illustration of the setup for calibrated TTA-UCL measurement under precisely controlled temperature. c The thermal sensitivity of TTA-Nd-NPs in aqueous solution. The spectra were measured with the excitation of coupled $635 \mathrm{~nm}\left(100 \mathrm{~mW} \mathrm{~cm}^{-2}\right)$ and $808 \mathrm{~nm}\left(100 \mathrm{~mW} \mathrm{~cm}^{-2}\right)$ laser. Inset: the integrated emission intensities of TTA-UCL (Em l, green line) and Reference signals (Em II, red line) as functions of temperature. $\mathbf{d}$ The concentration effects on the ratiometric probing method (blue line) and the intensity-based probing method (green line) at room temperature

method for TTA system. Furthermore, the $\mathrm{pH}$ factor that varied in different situations and tissues was also investigated, and the results under $\mathrm{pH}$ of 5.0-8.0 were almost uniform (Supplementary Figure 15). Moreover, the TTA-Nd-NPs probe was stable and with good reversibility (Supplementary Figure 16). These properties made the TTA-Nd-NPs suitable for temperature imaging in vivo without interference from the biological environment.

Ratiometric thermometry in vivo based on TTA-Nd-NPs. The standard curve of TTA-Nd-NPs for practical temperature evaluation in vivo was calibrated in the bioimaging system (Fig. 4a) ${ }^{17,48}$. The simulated condition in tissue phantom was an analogy of the subcutaneous injection in vivo (Supplementary Figure 17), and the data points was fitted as a following equation with $R^{2>0.999,}$ Ratio $\left(I_{\text {TTA-UCL }} / I_{\text {Reference }}\right)=0.00375 T^{2}$ $-2.12032 T+300.47099$ (Fig. $4 \mathrm{~b}$ ). The applicability of this calibration curve was then verified in the thermometry of a living mouse (Supplementary Figure 18). Actually, the TTA-Nd-NPs could reveal subcutaneous temperature in situ without physical contact, which was superior to the classical thermocouple that required skin puncture. Accordingly, the curve of thermal sensitivity $\left[S_{\mathrm{R}}=(\mathrm{dRatio} / \mathrm{d} T) /\right.$ Ratio] was shown in Supplementary Figure $19^{1,47,49,50}$. Based on these results, the thermal sensitivity was up to $\sim 7.1 \% \mathrm{~K}^{-1}$ with a high resolution of $\sim 0.1 \mathrm{~K}$, which represent the best results among all the thermometers developed for in vivo thermometry to date (Supplementary Table 1$)^{51}$.
Frankly, the weakness of this excellent TTA-Nd-NPs thermometer was revealed as the moderate penetration depth of green UCL signal (Supplementary Figure 20). Therefore, a thermosensitive TTA-UC system working in the NIR domain could be an attractive breakthrough for future applications in deep tissues. The NIR emissive sensitizer severing as reference could also simplify the systems in configuration ${ }^{46,47}$.

Furthermore, the possible applications of our thermometer in vivo was explored. Generally, inflammation is interrelated with the physiological disorder in biological system which may cause temperature-related variations $4,52,53$. The inflammatory phenomena has been demonstrated in distinct processes, such as the ischemia symptoms, physical trauma, microbial infection, and drug induction ${ }^{4,52,53}$. Previously, the nanothermometry results revealed that the inflammatory process in ischemia experiments could lead to distinctive thermal dynamics in mice ${ }^{4}$. With these in mind, we intended to establish a chemically-induced arthritis with carrageenan and to monitor the inflammation-based temperature fluctuations directly (Supplementary Figure 21). Notably, we demonstrated that the inflammation in arthritis model was time-dependent and was accompanied with temperature variations (Supplementary Figure 22). To verify the protocol feasiblity, the possible influence of excitation laser on thermometry was also investigated ${ }^{54}$. Indeed, the low-level laser exposure in the present study could keep its influence on thermometry at a minimum (Supplementary Figure 23). These observations proved 

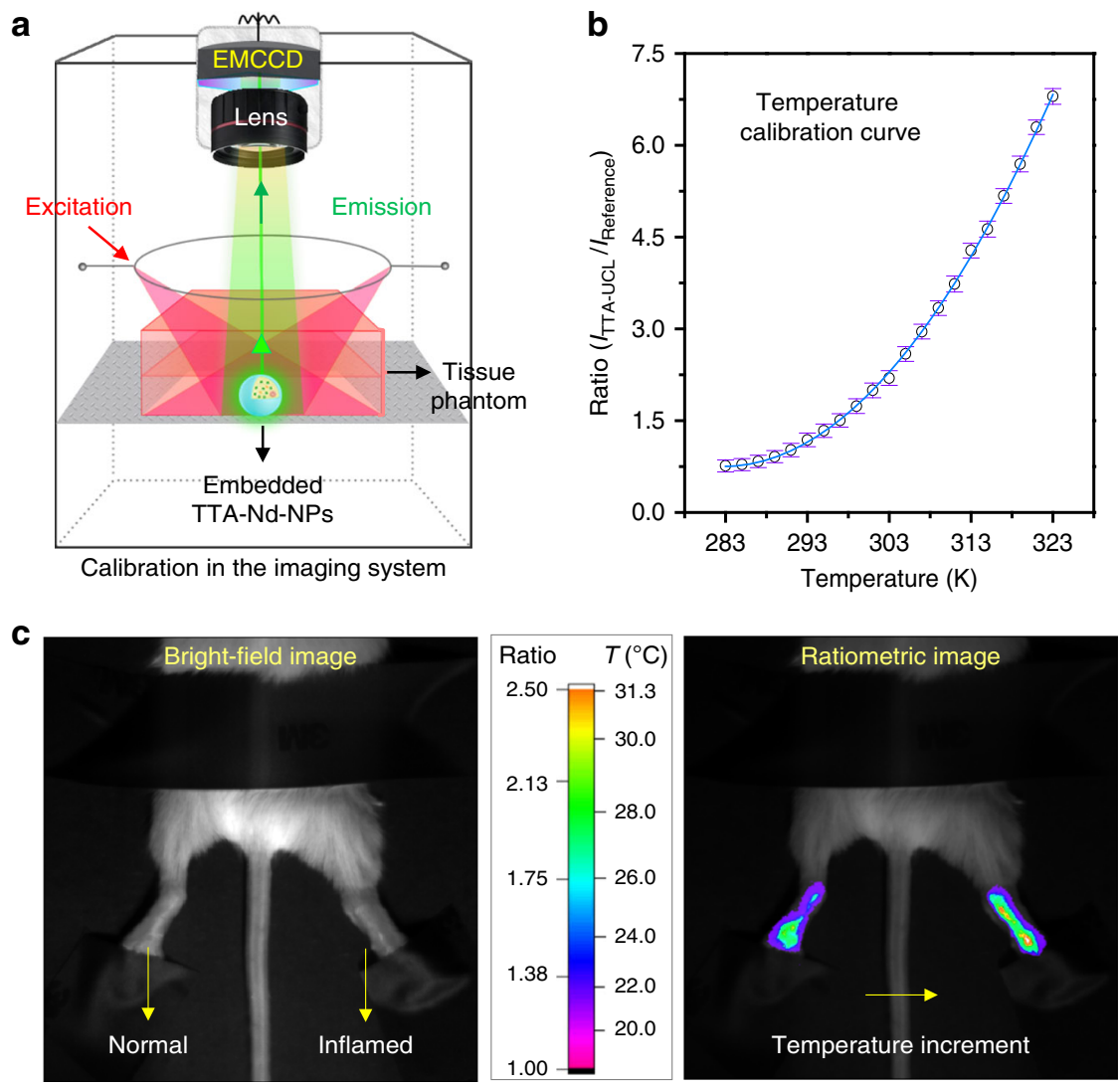

Fig. 4 Ratiometric thermometry in vivo. a Schematic illustration of the setup for ratiometric thermometry in vivo. b The standard curve for temperature evaluation in vivo measured with TTA-Nd-NPs probe in tissue phantom. $\mathbf{c}$ Ratiometric thermometry based on TTA-Nd-NPs in an inflammatory mode. The bright-filed image showed arthritis in the swollen right leg of a Kunming mouse stimulated with carrageenan (1 wt $\%$, $100 \mu \mathrm{L})$ while the left leg was normal as control. The ratiometric image revealed the luminescent ratios and evaluated temperature distributions in the two legs. The TTA-UCL and reference signals were acquired in channel I (485-575 nm) and channel II (980-1300 nm) of the in vivo imaging system, respectively

that our probe was promising for thermometry in the inflammation model.

Finally, the TTA-Nd-NPs based thermometer was tested for thermometry in the inflammatory mode. Herein, mild arthritis in the right leg of a mouse was induced with carrageenan while the other leg of the mouse was left normal as the control. As the arthritis model was established, a slight swell on the right leg of the mice was observed (Fig. 4c). The TTA-Nd-NPs based thermometer was then used to evaluate the specific temperature variations. As shown in Fig. 3c, different luminescent ratios between the two legs were clearly revealed. The ratiometric thermometry gave a clear map of temperature distributions and variations. According to the standard curve, the temperature of the arthritis area was higher than that of the normal area with an average difference of $\sim 0.9 \mathrm{~K}$. The result was in consistent with that achieved on a thermal camera (Supplementary Figure 24). Moreover, the observations also indicated that the TTA-Nd-NPs was little affected by the rigorous microenvironment in inflammation area where reactive oxygen species were relatively rich $^{55}$. Therefore, the TTA-Nd-NPs probe is capable of thermometry in vivo for monitoring the normal and abnormal temperature variations accurately.

\section{Discussion}

In the TTA system composed of annihilator and sensitizer dyad, an excited triplet sensitizer should diffuse to an annihilator nearby and participate in the triplet-triplet energy transfer (TTET) process to generate one triplet annihilator. Two triplet annihilators then approach each other through diffusion, and eventually annihilate to populate one singlet annihilator for the delayed TTA-UCL emission. Obviously, the diffusion is a positive factor for TTA-upconversion processes. TTA-UCL seems to become stronger at higher temperature, because the diffusion is positively correlated with temperature according to the Stokes-Einstein equation ${ }^{32}$. The multi-thermosensitive processes of TTA system could offer more scope for seeking a higher thermal sensitivity. Typically, TTA-upconversion was positively and highly temperature sensitive in case of blending the TTA chromophores in rubbery polymer chains ${ }^{24}$.

However, the conventional TTA systems were incapable for thermometry in vivo due to the two problems as below. One aspect of the matter, the TTA-temperature mechanism above was nonuniversal when rubbery polymers was not applicable as supporting medium. For example in the system employing phase-transition (gel-to-sol) matrix, the positive temperature-dependence of TTAUCL was no longer available ${ }^{27-29}$. The diffusion was approved to be a positive factor for TTA-UCL, but the negative effect of nonradiative deactivation was generally ignored. Indeed, the deactivation could lower the thermal sensitivity, or even led to a reverse response (e.g., positive at lower temperature and negative at higher temperature). Besides, without a temperature-independent standard internally, the conventional TTA system is also not qualified for thermometry in a complex biological environment ${ }^{42}$. Therefore, the development of TTA-based thermometry in vivo is still challenging.

The two key problems were both solved in this work. First of all, we have developed a highly thermosensitive TTA system by using the deactivation suppression strategy via molecular modification. The TTA-UCL was positively temperature-dependent over the 
physiological temperature range. Subsequently, the TTA system was encapsulated together with NIR-emissive but thermal-insensitive $\mathrm{Nd}^{3+}$ nanophosphors to construct the ratiometric TTA-Nd-NPs nanocomposite with high thermal sensitivity up to $\sim 7.1 \% \mathrm{~K}^{-1}$. The ratiometric TTA-UCL in TTA-Nd-NPs probe could provide stable signal output in spite of the fluctuant circumstances in living system. The potential use of TTA-Nd-NPs probe as a ratiometric thermometer in vivo was demonstrated by the accurate detection of temperature distribution in tissue and temperature increment caused by inflammation in mice. We believe the findings reported herein will assist in further research on the development of ratiometric thermometer in vivo.

\section{Methods}

Synthesis of BDM annihilator. Mesitaldehyde $(0.734 \mathrm{~g}, 4.95 \mathrm{mmol})$ and 3-ethyl2,4-dimethylpyrrole $(1.22 \mathrm{~g}, 9.9 \mathrm{mmol})$ were dissolved in dry $\mathrm{CH}_{2} \mathrm{Cl}_{2}(150 \mathrm{~mL})$ under nitrogen. One drop of trifluoroacetic acid (TFA) was added, and the resulting solution was stirred at room temperature for $6 \mathrm{~h}$ in the dark. Then, 2, 3Dichloro-5, 6-dicyanoquinone (DDQ, $1.12 \mathrm{~g}, 4.95 \mathrm{mmol}$ ) was added to the mixture, and the solution was stirred for additional $60 \mathrm{~min}$. The reaction mixture was then treated with triethylamine $(3 \mathrm{~mL})$ for $10 \mathrm{~min}$ and boron trifluoride etherate $(3.4 \mathrm{~mL})$ for another $2 \mathrm{~h}$. The dark brown solution was washed with water $(2 \times 40 \mathrm{~mL})$ and brine $(40 \mathrm{~mL})$, dried over anhydrous magnesium sulfate, and concentrated under reduced pressure. The crude product was purified by silica-gel flash column chromatography (elution with $10 \%$ EtOAc/petroleum ether) to yield $\mathrm{BDM}$ as a green crystal (yield 65\%). 1H-NMR $\left(400 \mathrm{MHz}, \mathrm{CDCl}_{3}\right) \delta 6.94(\mathrm{~s}, 2 \mathrm{H})$, $2.53(\mathrm{~s}, 6 \mathrm{H}), 2.34(\mathrm{~s}, 3 \mathrm{H}), 2.28-2.31(\mathrm{q}, 4 \mathrm{H}), 2.09(\mathrm{~s}, 6 \mathrm{H}), 1.29(\mathrm{~s}, 6 \mathrm{H}), 0.99-1.01$ (t, $6 \mathrm{H}$ ). Maldi-Tof/Tof-MS: calcd. $\left(\left[\mathrm{C}_{26} \mathrm{H}_{33} \mathrm{BF}_{2} \mathrm{~N}_{2}\right]^{+}\right) \mathrm{m} / z=422.2705$, found $\mathrm{m} / z$ $=422.2708$.

Synthesis of $\mathbf{N a Y F}_{\mathbf{4}}: \mathbf{5} \% \mathbf{N d}$ nanophosphors. In a typical procedure, $6 \mathrm{~mL}$ oleic acid and $15 \mathrm{~mL} 1$-octadecene, $0.95 \mathrm{mmol} \mathrm{YCl}$, and $0.05 \mathrm{mmol} \mathrm{NdCl}_{3}$ were added to a $100 \mathrm{~mL}$ three-necked flask. The mixture was heated to $160^{\circ} \mathrm{C}$ to form a clear solution under nitrogen flow. After the solution was cooled down to room temperature, $6 \mathrm{~mL}$ of methanol solution containing $2.5 \mathrm{mmol} \mathrm{NaOH}$ and $4 \mathrm{mmol}$ $\mathrm{NH}_{4} \mathrm{~F}$ was slowly added into the flask, and stirred for about $30 \mathrm{~min}$ at $80^{\circ} \mathrm{C}$. Then, the solution was degassed to remove residual water and oxygen at $120^{\circ} \mathrm{C}$ under vacuum for $15 \mathrm{~min}$. Subsequently, the solution was heated to $300^{\circ} \mathrm{C}$ and maintained for $1 \mathrm{~h}$ under nitrogen atmosphere. The solution was cooled naturally followed by an excessive amount of cyclohexane and ethanol were poured into. The resultant mixture was centrifugally separated, and the products were collected and washed with cyclohexane and ethanol for three times.

Preparation of TTA-Nd-NPs. BDM and PtTPBP were dissolved in nujol (concentrations of BDM and PtTPBP were $2 \times 10^{-3} \mathrm{~mol} \mathrm{~L}^{-1}$ and $2 \times 10^{-4} \mathrm{~mol} \mathrm{~L}^{-1}$, respectively) before $\mathrm{Nd}^{3+}$ nanophosphors $\left(15 \mathrm{mg} \mathrm{mL}^{-1}\right)$ were added in to get stock A. And BSA was dissolved in deionized water to get stock B (concentration of BSA was $1.5 \mathrm{mg} \mathrm{mL}^{-1}$ ). Then, $1 \mathrm{~mL}$ stock A and $20 \mathrm{mg}$ L-ascorbyl 6-palmitate were added into $100 \mathrm{~mL}$ stock B. The mixture was pre-emulsified at room temperature using an ultrasonic (Sonics VC750, Sonics \& Materials, Inc.) for $10 \mathrm{~min}$, and then was immediately emulsified using a high pressure nano homogenizer machine (FB110Q, LiTu Mechanical equipment Engineering Co., Ltd.) at 900 bar for $20 \mathrm{~min}$. The emulsion was heated at $90^{\circ} \mathrm{C}$ for $1 \mathrm{~h}$. Then, the emulsion was filtrated with $0.8 \mu \mathrm{m}$ membrane to obtain sterile emulsion. Finally, the uniform nanoparticles were obtained after gradient centrifugation. The other details of materials were shown in the Supplementary Information.

The measurement of absolute quantum efficiency. In the experiment, the TTANd-NPs material was dispersed in water at room temperature. The solution was then filled in a transparent quartz cuvette. The absolute quantum efficiency of BDM \& PtTPBP in TTA-Nd-NPs was measured with Hamamatsu instrument (C13532-12 Quantaurus-QY plus). A 635-nm laser with power density of $100 \mathrm{~mW}$ $\mathrm{cm}^{-2}$ was used as excitation light source. The absolute quantum efficiency of $\mathrm{NaYF}_{4}: 5 \% \mathrm{Nd}$ in TTA-Nd-NPs was measured with Photon Technology International instrument (PTI QM-40). An 808-nm laser with power density of $100 \mathrm{~mW}$ $\mathrm{cm}^{-2}$ was used as excitation light source. Light integrating spheres serving as accessory devices of the two instruments were used in the measurements.

The biocompatibility of TTA-Nd-NPs probe. The biocompatibility of TTA-NdNPs material was studied by using the standard methyl thiazolyl tetrazolium (MTT) assay. Hela cells $\left(1 \times 10^{4}\right.$ per well) were pre-cultured in the 96 -well plate. Subsequently, TTA-Nd-NPs at a series of concentrations $(0.05,0.1,0.15,0.3,0.5$, $1.0,1.5$, and $3.0 \mathrm{mg} \mathrm{mL}^{-1}$ ) were added to the wells while DMEM as the negative control group. The cells were incubated under $5 \% \mathrm{CO}_{2}$ at $37^{\circ} \mathrm{C}$ for $24 \mathrm{~h}$. After the MTT solution was added to each well, the cells were incubated for another $4 \mathrm{~h}$.
Then, an enzyme-linked immunosorbent assay reader (infinite M200, Tecan, Austria) was used to measure the cell viability.

Bioimaging and temperature monitoring in vivo. The animal procedures were in accordance with the guidelines of the Institutional Animal Care and Use Committee, Fudan University. UCL imaging in vivo was performed with an in vivo imaging system designed by our group, using CW 638\&808-nm laser (Changchun fs-optics Co., China) as the excited source and an Andor DU897 EMCCD as the signal collector. The TTA-UCL and reference signals were acquired in channel I $(485-575 \mathrm{~nm})$ and channel II $(980-1300 \mathrm{~nm})$ of the in vivo imaging system, respectively. In consideration of the effect of excitation laser on small animal, excessive laser irradiation (e.g., continuous exposure under intense laser for long periods) should be avoided ${ }^{54}$. Excitation laser with low power density

$\left(100 \mathrm{~mW} \mathrm{~cm}^{-2}\right)$ was used to minimize the laser-induced stress and damage. The duration of laser excitation at a time was less than $10 \mathrm{~s}$. The time was sufficient for acquiring images but not long enough to accumulate appreciable heat $(<0.1 \mathrm{~K})$. In some experiments, an IR thermal camera (FLIR E40) was used to imaging the temperature distributions on the surface of the mice.

Data availability. The data that support the findings of this study are available from the corresponding authors upon reasonable request.

Received: 5 February 2018 Accepted: 12 June 2018

Published online: 12 July 2018

\section{References}

1. Brites, C. D. S. et al. Thermometry at the nanoscale. Nanoscale 4, 4799-4829 (2012)

2. Jaque, D. \& Vetrone, F. Luminescence nanothermometry. Nanoscale 4, 4301-4326 (2012)

3. Wang, X. D., Wolfbeis, O. S. \& Meier, R. Luminescent probes and sensors for temperature. Chem. Soc. Rev. 42, 7834-7869 (2013).

4. Ximendes, E. C. et al. In vivo ischemia detection by luminescent nanothermometers. Adv. Helthc. Mater. 6, 1601195 (2017)

5. Somero, G. N. Proteins and temperature. Annu. Rev. Physiol. 57, 43-68 (1995).

6. Mackowiak, P. A. Concepts of fever. Arch. Intern. Med. 158, 1870-1881 (1998).

7. Zohar, O. et al. Thermal imaging of receptor-activated heat production in single cells. Biophys. J. 74, 82-89 (1998).

8. Wang, C. L. et al. Determining intracellular temperature at single-cell level by a novel thermocouple method. Cell Res. 21, 1517-1519 (2011).

9. Yang, J. M., Yang, H. \& Liu, L. W. Quantum dot nano thermometers reveal heterogeneous local thermogenesis in living cells. ACS Nano 5, 5067-5071 (2011).

10. Okabe, K. et al. Intracellular temperature mapping with a fluorescent polymeric thermometer and fluorescence lifetime imaging microscopy. Nat. Commun. 3, 705 (2012).

11. Auzel, F. Upconversion and anti-stokes processes with $\mathrm{f}$ and $\mathrm{d}$ ions in solids. Chem. Rev. 104, 139-174 (2004).

12. Wang, F. \& Liu, X. G. Recent advances in the chemistry of lanthanide-doped upconversion nanocrystals. Chem. Soc. Rev. 38, 976-989 (2009).

13. Zhou, J., Liu, Q., Feng, W., Sun, Y. \& Li, F. Y. Upconversion luminescent materials: advances and applications. Chem. Rev. 115, 395-465 (2015).

14. Jaque, D. et al. Fluorescent nanothermometers for intracellular thermal sensing. Nanomedicine 9, 1047-1062 (2014).

15. He, D. et al. Optical temperature sensing properties of $\mathrm{Yb}^{3+}-\mathrm{Er}^{3+}$ co-doped NaLnTiO4 ( $\mathrm{Ln}=\mathrm{Gd}, \mathrm{Y}$ ) up-conversion phosphors. RSC Adv. 5, 1385-1390 (2015).

16. Suo, H. et al. Thermometric and optical heating bi-functional properties of upconversion phosphor $\mathrm{Ba}_{5} \mathrm{Gd}_{8} \mathrm{Zn}_{4} \mathrm{O}_{21}: \mathrm{Yb}^{3+} / \mathrm{Tm}^{3+}$. J. Mater. Chem. C. 3, 7379-7385 (2015)

17. Zhu, X. J. et al. Temperature-feedback upconversion nanocomposite for accurate photothermal therapy at facile temperature. Nat. Commun. 7, 10437 (2016).

18. Rodríguez-Sevilla, P. et al. Thermal scanning at the cellular level by an optically trapped upconverting fluorescent particle. Adv. Mater. 28, $2421-2426$ (2016).

19. Singh-Rachford, T. N. \& Castellano, F. N. Photon upconversion based on sensitized triplet-triplet annihilation. Coord. Chem. Rev. 254, 2560-2573 (2010).

20. Simon, Y. C. \& Weder, C. Low-power photon upconversion through triplet-triplet annihilation in polymers. J. Mater. Chem. 22, 20817-20830 (2012)

21. Zhao, J. Z., Wu, W. H., Sun, J. F. \& Guo, S. Triplet photosensitizers: from molecular design to applications. Chem. Soc. Rev. 42, 5323-5351 (2013). 
22. Yanai, N. \& Kimizuka, N. Recent emergence of photon upconversion based on triplet energy migration in molecular assemblies. Chem. Commun. 52, 5354-5370 (2016).

23. Filatov, M. A., Baluschev, S. \& Landfester, K. Protection of densely populated excited triplet state ensembles against deactivation by molecular oxygen. Chem. Soc. Rev. 45, 4668-4689 (2016).

24. Singh-Rachford, T. N., Lott, J., Weder, C. \& Castellano, F. N. Influence of temperature on low-power upconversion in rubbery polymer blends. J. Am Chem. Soc. 131, 12007-12014 (2009).

25. Borisov, S. M., Larndorfer, C. \& Klimant, I. Triplet-triplet annihilation-based anti-stokes oxygen sensing materials with a very broad dynamic range. $A d v$. Funct. Mater. 22, 4360-4368 (2012).

26. Goudarzi, H. \& Keivanidis, P. E. Triplet-triplet annihilation-induced upconverted delayed luminescence in solid-state organic composites: monitoring low-energy photon up-conversion at low temperatures. J. Phys. Chem. C. 118, 14256-14265 (2014)

27. Duan, P. F., Yanai, N., Nagatomi, H. \& Kimizuka, N. Photon upconversion in supramolecular gel matrixes: spontaneous accumulation of light-harvesting donor-acceptor arrays in nanofibers and acquired air stability. J. Am. Chem. Soc. 137, 1887-1894 (2015).

28. Massaro, G., Hernando, J., Ruiz-Molina, D., Roscini, C. \& Latterini, L. Thermally switchable molecular upconversion emission. Chem. Mater. 28, 738-745 (2016).

29. Thévenaz, D. C. et al. Thermoresponsive low-power light upconversion polymer nanoparticles. Mater. Horiz. 3, 602-607 (2016).

30. Askes, S. H. C., Brodie, P., Bruylants, G. \& Bonnet, S. Temperature dependence of triplet-triplet annihilation upconversion in phospholipid membranes. J. Phys. Chem. B 121, 780-786 (2017).

31. Hisamitsu, S., Yanai, N. \& Kimizuka, N. Photon-upconverting ionic liquids: effective triplet energy migration in contiguous ionic chromophore arrays. Angew. Chem. Int. Ed. 54, 11550-11554 (2015).

32. Ye, C. Q., Zhou, L. W., Wang, X. M. \& Liang, Z. Q. Photon upconversion: from two-photon absorption (TPA) to triplet-triplet annihilation (TTA). Phys. Chem. Chem. Phys. 18, 10818-10835 (2016).

33. Askes, S. H. C. et al. Imaging upconverting polymersomes in cancer cells: biocompatible antioxidants brighten triplet-triplet annihilation upconversion. Small 12, 5579-5590 (2016).

34. Cerón, E. N. et al. Hybrid nanostructures for high-sensitivity luminescence nanothermometry in the second biological window. Adv. Mater. 27, 4781-4787 (2015).

35. Smith, A. M., Mancini, M. C. \& Nie, S. Second window for in vivo imaging. Nat. Nanotechnol. 4, 710-711 (2009).

36. Robinson, J. T. et al. In vivo fluorescence imaging in the second near-infrared window with long circulating carbon nanotubes capable of ultrahigh tumor uptake. J. Am. Chem. Soc. 134, $10664-10669$ (2012).

37. Singh-Rachford, T. N., Haefele, A., Ziessel, R. \& Castellano, F. N. Boron dipyrromethene chromophores: next generation triplet acceptors/annihilators for low power upconversion schemes. J. Am. Chem. Soc. 130, 16164-16165 (2008).

38. Liu, Q. et al. A general strategy for biocompatible, high-effective upconversion nanocapsules based on triplet-triplet annihilation. J. Am. Chem. Soc. 135, 5029-5037 (2013).

39. Kim, J. H., Deng, F., Castellano, F. N. \& Kim, J. H. Red-to-blue/cyan/green upconverting microcapsules for aqueousand dry-phase color tuning and magnetic sorting. ACS Photonics 1, 382-388 (2014).

40. Xu, K. J., Zhao, J. Z., Cui, X. N. \& Ma, J. Photoswitching of triplet-triplet annihilation upconversion showing large emission shifts using a photochromic fluorescent dithienylethene-Bodipy triad as a triplet acceptor/ emitter. Chem. Commun. 51, 1803-1806 (2015).

41. Würth, C., Grabolle, M., Pauli, J., Spieles, M. \& Resch-Genger, U. Relative and absolute determination of fluorescence quantum yields of transparent samples. Nat. Protoc. 8, 1535-1550 (2013).

42. Baffou, G., Rigneault, H., Marguet, D. \& Jullien, L. A critique of methods for temperature imaging in single cells. Nat. Methods 11, 899-901 (2014).

43. Monguzzi, A., Mezyk, J., Scotognella, F., Tubino, R. \& Meinardi, F. Upconversion-induced fluorescence in multicomponent systems: steady-state excitation power threshold. Phys. Rev. B 78, 195112 (2008).

44. Rocha, U. et al. Subtissue thermal sensing based on neodymium-doped $\mathrm{LaF}_{3}$ nanoparticles. ACS Nano 7, 1188-1199 (2013).

45. Carrasco, E. et al. Intratumoral thermal reading during photo-thermal therapy by multifunctional fluorescent nanoparticles. Adv. Funct. Mater. 25, 615-626 (2015).
46. Ximendes, E. C. et al. In vivo subcutaneous thermal video recording by supersensitive infrared nanothermometers. Adv. Funct. Mater. 27, 1702249 (2017).

47. Ximendes, E. C. et al. Unveiling in vivo subcutaneous thermal dynamics by infrared luminescent nanothermometers. Nano. Lett. 16, 1695-1703 (2016).

48. Grand, A. M. D. et al. Tissue-like phantoms for near-infrared fluorescence imaging system assessment and the training of surgeons. J. Biomed. Opt. 11, 014007 (2006).

49. Wade, S. A., Collins, S. F. \& Baxter, G. W. Fluorescence intensity ratio technique for optical fiber point temperature sensing. J. Appl. Phys. 94, 4743-4756 (2003)

50. Benayas, A. et al. $\mathrm{PbS} / \mathrm{CdS} / \mathrm{ZnS}$ quantum dots: a multifunctional platform for in vivo near-infrared low-dose fluorescence imaging. Adv. Funct. Mater. 25 6650-6659 (2015).

51. del Rosal, B., Ximendes, E., Rocha, U. \& Jaque, D. In vivo luminescence nanothermometry: from materials to applications. Adv. Opt. Mater. 5, 1600508 (2017).

52. Brenner, M., Braun, C. \& Gulko, P. S. Thermal signature analysis as a novel method for evaluating inflammatory arthritis activity. Ann. Rheum. Dis. 65 , 306-311 (2006).

53. Sanchez, B. M. et al. Use of a portable thermal imaging unit as a rapid, quantitative method of evaluating inflammation and experimental arthritis. J. Pharmacol. Toxicol. Methods 57, 169-175 (2008).

54. del Rosal, B., Villa, I., Jaque, D. \& Sanz-Rpdríguez, F. In vivo autofluorescence in the biological windows: the role of pigmentation. J. Biophotonics. 9, 1059-1067 (2016).

55. Chapple, I. L. C. Reactive oxygen species and antioxidants in inflammatory diseases. J. Clin. Periodontol. 24, 287-296 (1997).

\section{Acknowledgements}

The authors thank the National Natural Science Foundation of China (21527801, 21231004, 21701109, and 21722101), the National Key R\&D Program of China (Grant 2017YFA0205100 and 2016YFC130310), the National Basic Research Program of China (2015CB931800), and Shanghai Sci. Tech. Comm. (15QA1400700) for financial support.

\section{Author contributions}

The manuscript was written by M.X, W.F., and F.L. The experiment and analysis were carried out by M.X., X.Z., Q.S., W.Y., C.C., Q.W., and X.Z. The experimental work and the manuscript were supervised by W.F. and F.L.

\section{Additional information}

Supplementary Information accompanies this paper at https://doi.org/10.1038/s41467018-05160-1.

\section{Competing interests: The authors declare no competing interests.}

Reprints and permission information is available online at http://npg.nature.com/ reprintsandpermissions/

Publisher's note: Springer Nature remains neutral with regard to jurisdictional claims in published maps and institutional affiliations.

\footnotetext{
Open Access This article is licensed under a Creative Commons BY Attribution 4.0 International License, which permits use, sharing,
adaptation, distribution and reproduction in any medium or format, as long as you give appropriate credit to the original author(s) and the source, provide a link to the Creative Commons license, and indicate if changes were made. The images or other third party material in this article are included in the article's Creative Commons license, unless indicated otherwise in a credit line to the material. If material is not included in the article's Creative Commons license and your intended use is not permitted by statutory regulation or exceeds the permitted use, you will need to obtain permission directly from the copyright holder. To view a copy of this license, visit http://creativecommons.org/ licenses/by/4.0/.
}

(C) The Author(s) 2018 